\title{
Quark mean-field theory and consistency with nuclear matter
}

\author{
Jishnu Dey* and Lauro Tomio ${ }^{\dagger}$ \\ Instituto de Física Teórica, Universidade Estadual Paulista, Rua Pamplona 145, 01405 São Paulo, Brazil \\ Mira Dey \\ Department of Physics, Maulana Azad College, Calcutta 700013, India \\ T. Frederico $\ddagger$ \\ Institute for Nuclear Theory, Department of Physics, FM-15, University of Washington, Seattle, Washington 98195
}

(Received 21 March 1991)

\begin{abstract}
$1 / N_{c}$ expansion in QCD (with $N_{c}$ the number of colors) suggests using a potential from meson sector (e.g., Richardson) for baryons. For light quarks a $\sigma$ field has to be introduced to ensure chiral symmetry breaking $(\chi \mathrm{SB})$. It is found that nuclear matter properties can be used to pin down the $\chi \mathrm{SB}$ modeling. All masses, $M_{N}, m_{\sigma}, m_{\omega}$, are found to scale with density. The equations are solved self-consistently.
\end{abstract}

Low-energy physics is essentially controlled by the Goldstone particle, i.e., the pion. The relevant parameter is the pion decay constant, $f_{\pi}$. This is related to the quark condensate $\langle\bar{q} q\rangle$ through the Weinberg sum rule. In the QCD sum-rule approach also, the nucleon mass is determined predominantly by the odd-dimensional operator $\langle\bar{q} q\rangle$ (Ioffe [1], Reinders, Rubinstein, and Yazaki [2]). At higher density the condensate decreases in magnitude (Bailin, Cleymans, and Scadron [3], Dey, Dey, and Ghose [4]) and $f_{\pi}$ also decreases correspondingly (Dey and Dey [5]). This results in changes in the nucleon property, for example, in the increase in the radius expected from the European Muon Collaboration experimental data through rescaling (Close, Roberts, and Ross [6]). In the formalism of relativistic Hartree-Fock theory (Dey, Dey, and Le Tourneux [7]), justified by the large $N_{c}$ theory of t'Hooft [8] and Witten [9], one gets a weakening of the confinement (Dey et al. [10]) at higher density. If one uses the potential due to Richardson [11], this means a decrease in $\Lambda$, the only parameter present in the interaction. We wish to recall that for light quarks one has to use a running quark mass $m(r)$ to get correct nucleon radius and other properties [7].

Relativistic description of nuclear structure and reactions within quantum hadrodynamics (QHD) has been greatly developed during the last several years (Serot and Walecka [12], Celenza, Rozenthal, and Shakin [13]). Can one show the compatibility of this model with the meanfield quark model? In a crude way this was done by Guichon [14] and Frederico et al. [15] where they assumed that there is a scalar $\sigma$ and a vector $\omega$ field coupled to the quark. As clearly stated by Guichon, this is a very strong assumption since neither the $\sigma$ nor the $\omega$ are fundamental at the quark level. One can think of the running quark mass $m(x)$ as being due to a $\sigma$ field. There are two problems in doing this. First one has to take care of the Goldstone pion since one is breaking chiral symmetry. This is hard to do since the pion-quark coupling is a highly nonlinear one and only in the Zahed model [16] in lower dimension can one treat this exactly. But we need not worry about this here since we can introduce the well-established one-pion-exchange potential (OPEP) at the QHD level in nuclear matter and thus correct for the deficiency of the quark model. In some models, pions may indeed couple to the quarks themselves, but we do not consider such models. The problem is that the pions have a dual character, being Goldstone particles as well as quark-antiquark composites; their role in QCD-inspired models is still ambiguous. For the present we prefer to ignore the pion-quark interaction and replace this by the nucleon-pion interaction, which does not introduce any additional parameters in the theory. The energy due to the OPEP in nuclear matter has been estimated in second-order perturbation theory by Cenni, Conte, and Dillon [17] using wave functions in nuclear matter derived from the Reid soft-core potential [18]. This is almost model independent since the OPEP tail is the same in all modern nucleon-nucleon interactions. And the second-order OPEP contribution is insensitive to the finer details of the nuclear matter wave function-it only depends on the wound in the wave function in a crude sort of way. One can see that the error due to this cannot be very large even if pions couple to the quarks themselves as Goldstones, since the coupling must be cut off for a radius of around $0.35 \mathrm{fm}$ or so. This is because from deep-inelastic scattering we know that at short distances the quarks are free and massless. In fact, in models like the $\sigma-\pi$ soliton bag this restoration of chiral symmetry at short distance is hard to achieve. In some sense the Reid soft-core wound cuts off the pion from the nucleon, and therefore also from the quarks at about this distance, since in the nuclear-matter wave function, the nucleon is taken to be pointlike, apart from this wave-function effect. At the present moment, exact treatment of the nucleon-nucleon interaction with pions in the quark picture, is an insoluble problem. The $\sigma$ fits in nicely with the running quark mass, but again there is problem with the vector mesons. In the Walecka model 
there is the $\omega$ meson and this also we couple to the nucleon rather than the quarks themselves. Now, of course, extra parameters are introduced and the purpose of the paper is to report that the nuclear matter constraints are enough to uniquely determine these parameters.

We will next look at the problem from the QCD point of view. Starting from the action for a system of interacting quarks and gluons one can obtain, after a series of approximations, a Dirac Hamiltonian with a two-body static potential. It was shown by t'Hooft [8] that such a classical (as opposed to field theoretic where $\bar{q} q$ loops essentially introduces infinite degrees of freedom) two-body interaction may be derived by summing all the gluon loops that one can draw on a plane. Witten [9] further showed that this interaction, which is appropriate for the meson (essentially a two-body system), can also be used in the mean-field approximation for a baryon in the same order. Present-day techniques do not permit summing up all the planar gluon diagrams which would yield such a potential unambiguously. As an alternative one can borrow a potential from the meson calculation, for example, that of Crater and van Alstine [19] mentioned before and test it for a baryon (Dey, Dey, and Le Tourneux [7]). The potential used in this case is due to Richardson [11] and it passes the test very well. For the sake of completeness we present this potential here:

$$
V(r)=\frac{6 \pi}{4} \frac{\lambda(1) \lambda(2)}{33-2 N_{f}} \Lambda\left[\Lambda r-\frac{f(\Lambda r)}{\Lambda r}\right],
$$

where we have the scalar product of the color SU(3) matrices $\lambda s$ for the two interacting quarks, $N_{f}$ is the number of flavors taken to be three, and

$$
f(y) \equiv 1-4 \int_{1}^{\infty} \frac{d q}{q} \frac{\exp (-q y)}{\ln \left(q^{2}-1\right)+\pi^{2}} .
$$

The action of a system of interacting quarks and gluons can be written as

$$
S=S_{\text {gluon }}+\int d^{4} x\left[\bar{q}(i \not \partial-m) q+j_{\mu}^{a} A^{a \mu}\right],
$$

where $S_{\text {gluon }}$ denotes, collectively, the action of gluons, the gauge-fixing terms and the action of the unphysical or ghost fields, $A$ the field potential, while $j_{\mu}^{a}$ is the quark current

$$
j_{\mu}^{a}=\frac{g}{2} \bar{q} \gamma_{\mu} \Lambda^{a} q
$$

The connected Green's functions of gluons are generated by the functional

$e^{i W(j)}=\int d \mu[A] \exp \left[i\left(S_{\text {gluon }}+\int j_{v}^{a} A^{a v} d^{4} x\right)\right]$

where $d \mu$ includes the ghost fields. The full generating functional is given by

$$
Z=\int d \mu[A][d \bar{q} d q] e^{i S_{\mathrm{QCD}}}
$$

and using (3) one can formally integrate out the gluons and ghosts and write

$$
Z=\int[d \bar{q} d q] \exp \left[i \int d^{4} x \bar{q}(i \not \partial-m) q+W(j)\right],
$$

thus obtaining an effective action for the quarks:

$$
S_{\mathrm{eff}}=\int d^{4} x\left[\bar{q}(i \not \partial-m) q-\frac{1}{2} \int j^{a \mu}(x) V_{\mu \nu}^{a b}(x, y) j^{b v}(y) d^{4} y-\frac{1}{6} \int j^{a \mu}(x) V_{\mu \nu \rho}^{a b c}(x, y, z) j^{b v}(y) j^{c \rho}(z) d^{4} y d^{4} z-\cdots\right],
$$

where the $V$ 's are connected Green's functions. This equation is an infinite expansion and it is absolutely essential to have a truncation scheme if we want to extract meaningful numbers. For $N_{c}$-quark systems like the baryon, the $1 / N_{c}$ expansion provides such a scheme. This can be seen by going back to the formalism of canonical quantization and considering $S_{\text {eff }}$ as a function of the field operators $q$ and $\bar{q}$. For an $N_{c}$-body baryon, the expectation value of the $N$ currents $\left(N>N_{c}\right)$ that are contracted with $V_{\mu_{1}}^{a_{1} \cdots \mu_{N}}$ involves

$$
\left\langle N_{c} q^{\prime} s\right| \bar{q} q \cdots \bar{q} q(N \text { times })\left|N_{c} q^{\prime} s\right\rangle \approx \Psi_{N_{c}}^{*}\left(1, \cdots, N_{c}\right)\langle 0| \bar{q} q \cdots \bar{q} q\left(N-N_{c} \text { times }\right)|0\rangle \Psi_{N_{c}}\left(1, \cdots, N_{c}\right)
$$

Now the factor $\langle 0| \bar{q} q \cdots \bar{q} q\left(N-N_{c}\right.$ times $)|0\rangle$ corresponds to the production of virtual $\bar{q} q$ pairs and quark loops. It is suppressed by $1 / N_{c}$ and all terms involving more than $N_{c}$ currents can be dropped in a similar manner. In spite of this restriction one cannot actually compute even the two-point Green's function to all orders. One therefore takes the static limit and use for $V_{00}$ a standard vector potential like Richardson's, for example. For light $(u, d)$ quarks one has to split the potential into a vector and a scalar part arbitrarily to take care of the chiral symmetry breaking [7]. An alternative has been tried recently by Dey et al. [20], where forms are taken for the mass following Shuryak [21] or Brevik [22]. We will discuss the form given by Shuryak briefly (details may be found in pp. 123-126 of his book). This gives

$$
m_{\text {quark }}(x)=-4 \pi^{2} x^{2}\langle\bar{\Psi} \Psi\rangle
$$

where $\langle\bar{\Psi} \Psi\rangle \approx(-255 \mathrm{MeV})^{3}$ and the Eq. (10) is valid up to about $0.35 \mathrm{fm}$ from which point the quark mass is taken to be flat and constant. The interesting thing is that the form of the Hartree-Fock potential is not sensitively dependent on the form of the mass chosen, i.e., there is not too much difference between the Shuryak [21] form 
given above and the form given by Brevik [22]. In a sense this effective mass term is due to the $\bar{q} q$ terms of Eq. (9) taken in a heuristic manner. So it is comforting to know that while the quark mass has to be taken in a form like in Eq. (10), the results are not sensitive on its precise form chosen. It may be relevant at this point to recall that $\bar{q} q$ degrees of freedom may be more important in baryons than people thought before. We refer to only two suggestions in the current literature: (a) Jaffe [23] has suggested large coupling of the nucleon to the $\phi$ and (b) Preparata and Soffer [24] have suggested large coupling to $\eta^{\prime}$, both of which are present in the original analysis of Nagels, Rijken, and de Swart [25] and have been neglected in nuclear physics for a long time.

To return to the problem we recall that one cannot use a coordinate-dependent form for the $m(x)$ in a Lagrangian formalism, but has to introduce a field $\sigma(x)$ instead. This field is an effective field and its properties may therefore change with the ambient density. This relates the quark problem to the nuclear matter problem and it is possible to find consistent solutions for the nuclear binding-energy curve from this. One has to invoke a vector $\omega$ field also and, as stated by Guichon [14] and Frederico, Carlson, Rego, and Hussein [15] who first suggested this kind of treatment, these are not fundamental at the quark level. We find that if one couples this approach with the relativistic HF at the quark level, the stringent condition of matching the $\sigma(r)$ with the quark $\bar{\psi} \psi$ determines the energy-density function of the $\sigma U(\sigma)$, and mass of the $\sigma$ scales as $f_{\pi}$ with density. $U(\sigma)$ looks like that found in soliton bag models. We will elaborate on this interesting point. One can start with a parabolic form of $U$ for zero density but then finds that cubic and quartic terms in $\sigma$ have to be added. This is not unexpected since at high density if chiral symmetry is to be restored one must have a second minimum in $U(\sigma)$. This is not possible with a parabolic form since the $d U / d \sigma$ is then linear in $\sigma$. This type of nonlinearity has been known in the soliton bag model for a long time [26] but it is nice to find numerical analysis compelling one to similar solutions when the starting point is quite different.

One can fit nuclear matter in a satisfactory manner if one takes an $\omega$ mass which also scales with density like $f_{\pi}$ and takes a standard form for the one-pion-exchange potential contribution to nuclear matter (from, for example, Cenni, Conte, and Dillon [17]). This latter contribution from the OPEP is quite justified, since QCD sum rules predict essentially the correct value for the pionnucleon coupling constant (Reinders, Rubinstein, and Yazaki [2]) and this coupling constant has very little density dependence around normal nuclear matter density (Dey, Dey, and Ghose [4]). The scaling of the mass of $\sigma$ with density was expected by Brown and Rho [27] from the missing strength of the longitudinal electron response. It is interesting to find that the same behavior is essential to get a fit to nuclear matter starting from a quark mean-field model.

As stated before, one can identify $m(r)$ with a $\sigma$ field in the spirit of Friedberg and Lee model [26]. The difficulty is that one has to solve coupled equations for the $\sigma$ fields and the quark field $\psi$ as done by Goldflam and Wilets [26], but with one more complication. There is now a mean field obtained from two-body quark-quark potential self-consistently. We have

$$
[\alpha \cdot p+\beta g \sigma(r)] \psi=[\epsilon-v(r)] \psi,
$$

with

$$
\frac{d^{2} \sigma}{d r^{2}}+\frac{2}{r} \frac{d \sigma}{d r}-\frac{d U(\sigma)}{d \sigma}=3 g \bar{\psi} \psi
$$

and

$$
v(r)=\int d^{3} r^{\prime} \psi^{\dagger}\left(r^{\prime}\right) V\left(r-r^{\prime}\right) \psi\left(r^{\prime}\right),
$$

where $V\left(r-r^{\prime}\right)$ is the two-body quark-quark potential. $g$ is the quark- $\sigma$ coupling constant. The mean field $v(r)$ is totally confined inside the nucleon. $U(\sigma)$ is given by

$$
U(\sigma)=\frac{m_{\sigma}^{2}}{2}\left(\sigma-\sigma_{v}\right)^{2}+\frac{t_{2}}{3 \sigma_{v}}\left(\sigma-\sigma_{v}\right)^{3}+\frac{t_{3}}{4 \sigma_{v}^{2}}\left(\sigma-\sigma_{v}\right)^{4},
$$

where the scalar $\sigma$ field attains its vacuum expectation value $\sigma_{v}$ so that

$$
g \sigma_{v}=m_{q},
$$

$m_{q}$ being the constituent quark mass taken to be 300 $\operatorname{MeV}\left(1.5 \mathrm{fm}^{-1}\right)$. Observe that we wrote $U(\sigma)$ in such a way that $m_{\sigma}$ is the effective $\sigma$ mass:

$$
m_{\sigma}^{2}=\left(\frac{d^{2} U}{d \sigma^{2}}\right)_{\sigma=\sigma_{v}} .
$$

$g \sigma(r)$ can be fitted to an analytic form,

$$
g \sigma(r)=m(r)=m_{q}\left[1-\left(1+\alpha r+\alpha^{2} r^{2} / 3\right) \exp (-\alpha r)\right] .
$$

This form for the $\sigma$ field was reached by a step in the process of solving consistently the equations (11)-(14). We found this as the simple analytical solution that gives the best approach for the coupled equations, and can fit well the numerical results we obtain for each parameter $\Lambda$, corresponding to some specific nuclear density. The parameter $\Lambda$ of the Richardson potential was fitted to various nuclear densities, as explained in Refs. [11] and [20]. In Eq. (17), we let $\alpha$ be a parameter to be adjusted to have the consistency fulfilled. We have used a tedious but reliable process to obtain this part of the self-consistency. We have used a graphical package to plot the $\sigma$ field in order to adjust the analytical form to our numerical results, obtained from the Hartree-Fock calculation. This gives us a new input for the $\sigma$ field, until the consistency is reached. The parameter $\alpha$ we have obtained in this process was equal to $5 \mathrm{fm}^{-1}$, consistent with the model of Ref. [21].

It is possible that the quark mass may vary with density, but we found that if we incorporate such an effect directly in this stage of our approach, such procedure can face problems with double counting, and may be not compatible with the model. We mean "double counting" in the following sense: the effective mass has already in- 
corporated in it the effect of the $\sigma$ mean field, as we are assuming the $\sigma-\omega$ model for the nucleon propagation in nuclear matter. The constituent quark masses are components of the nucleon mass; and, in case we consider that also the constituent quark mass has the effect of the $\sigma$ field, such effect will be doubled in the effective mass of the nucleon. Then in the present formulation of our model we favor the first option; it means only the nucleon in the sense of the $\sigma-\omega$ model has an effective mass. We expect the possible variation with the density in the constituent quark mass will not change our results qualitatively. The formulation of such a model avoiding the double counting will be pursued in a future work.

With the form given in Eq. (17) we calculate the nucleon mass from the relativistic Hartree-Fock equation self-consistently [7]. The center-of-mass correction is also done as in Ref. [7]. The energy contribution of the $\sigma$ field to the nucleon mass is obtained by integrating $U(\sigma)$ and the kinetic-energy density $\left[\frac{1}{2}(\nabla \sigma)^{2}\right]$ of the $\sigma$ field. The resulting mass $M_{N}$ is a bit high but we do not worry about this since pion cloud correction could bring this down. Fortunately, the nuclear-matter binding is independent of this mass since this is subtracted out from the energy. As seen from this equation, for large $r, \sigma$ becomes a constant and a constant $\sigma$ is what is used in the Walecka model. The coupling with the nucleon is given by

$$
g_{\sigma N}=3 g \int d^{3} r \bar{\psi} \psi
$$

The $\omega$ field is introduced at this level following Serot and Walecka [13] so that the energy density of the nuclear matter is

$$
\begin{aligned}
\mathscr{E}= & \mathscr{E}_{\pi}+\frac{g_{\omega}^{2}}{2 m_{\omega}^{2}} \rho_{B}^{2}+\frac{1}{2}\left[\frac{m_{\sigma}}{g_{\sigma N}}\left(M_{N}-M^{*}\right)\right]^{2} \\
& +\frac{4}{(2 \pi)^{3}} \int_{0}^{k_{F}} d^{3} k \sqrt{k^{2}+M^{* 2}} \\
\equiv & \mathscr{E}_{\pi}+\mathscr{E}_{\omega}+\mathscr{E}_{s}+\mathscr{E}_{K} .
\end{aligned}
$$

Here $\rho_{B}$ is the nuclear-matter density, $k_{F}$ is the Fermi momentum, and $M^{*}$ is the effective nucleon mass in the medium to be found self-consistently from

$M^{*}=M_{N}-\left(\frac{g_{\sigma N}}{\pi m_{\sigma}}\right)^{2}\left[k_{F} E_{F}-M^{* 2} \ln \left(\frac{k_{F}+E_{F}}{M^{*}}\right)\right] M^{*}$,

where

$$
E_{F}=\sqrt{M^{* 2}+k_{F}^{2}}
$$

To obtain the saturation at $\rho_{B}$ equal to $\rho_{0}=(0.17$ $\left.\mathrm{fm}^{-3}\right) g_{\omega}$ is adjusted to

$$
E_{\omega}=\frac{243.5}{2 M_{N}^{2}} \rho_{B}
$$

The number used by Serot and Walecka [12] was 193.7, the experimental one, given in Ref. [14], being 251 is closer to 243.5 .
We would like to emphasize here the way the selfconsistency is obtained in this calculation. We solve the equations for $\psi$ and $\sigma$, Eqs. (11)-(17), using the Richardson potential [11] [see Eqs. (1) and (2)] for different values of the parameter $\Lambda$, for the corresponding nuclear densities. This parameter was fitted for different nuclear densities, as in Ref. [11] and [20], and we reproduce in Table I the corresponding variation. From this consistency we obtain the density dependence of the quark- $\sigma$ coupling constant $g$ and the corresponding coupling with the nucleon given by Eq. (18). Next we calculate the nucleon mass from the relativistic Hartree-Fock equation selfconsistently. We also have included the center-of-mass contribution as has been done in Ref. [7]. The $\sigma$ energy contribution to the nucleon mass $E_{\sigma}$ is added at this point, after integration of the density energies (kinetic and potential). After this we have the running nucleon mass $M_{N}$, that varies with the density, and the effective nucleon mass in the medium is obtained self-consistently through Eqs. (20) and (21). The energy density of the nuclear matter is obtained through Eq. (19) and the numerical constant present in the $\omega$ energy, Eq. (22), adjusts the saturation at the normal nuclear density.

In Table I for each density we list the corresponding $\Lambda$, the parameters of $U(\sigma)$, the quark- $\sigma$ coupling, and the nucleon- $\sigma$ coupling. In Table II, again for the same densities, we give the $\sigma$ contribution for the nucleon mass $E_{\sigma}$, the nucleon mass $M_{N}$, the self consistent mass $M^{*}$, the energy contributions to the binding and the final binding energy (BE) per nucleon. This final binding energy is obtained after summing all the energy contributions and subtracting the nucleon energy at zero density. A nice feature is that the saturation is obtained with a consistent set of parameters. Only a large $\sigma$ mass can give us the saturation in the right position and with approximately the correct amount of binding energy per nucleon. The relation connecting $t_{2}$ and $t_{3}$, given by $\left(t_{2} / 2 m_{\sigma}\right)^{2}$, ensures that we have just one minimum of the potential $U$ at $\sigma=\sigma_{v}$ and that the other two extremes are in the same position. We can also produce another minimum with a smaller value of $t_{3}$, but to keep the absolute minimum at $\sigma=\sigma_{v}$ one cannot change $t_{3}$ too much. The expressions for the energies $E_{\mu}$ are defined by

TABLE I. Density dependence of the parameters and coupling constants.

\begin{tabular}{lccccc}
\hline \hline$\rho_{B} / \rho_{0}$ & $\Lambda(\mathrm{MeV})$ & $m_{\sigma}(\mathrm{MeV})$ & $t_{2}\left(\mathrm{fm}^{-} 2\right)$ & \multicolumn{1}{c}{$g$} & $g_{\sigma N}$ \\
\hline 0 & 225 & 2565 & 0 & 13.418 & 19.861 \\
0.25 & 217 & 2488 & 15 & 12.844 & 19.733 \\
0.50 & 209 & 2389 & 27 & 12.157 & 19.110 \\
0.75 & 202 & 2293 & 37 & 11.529 & 18.659 \\
1.00 & 194 & 2197 & 46 & 10.919 & 18.213 \\
1.25 & 187 & 2118 & 53.5 & 10.402 & 17.854 \\
1.50 & 180 & 2025 & 59 & 9.806 & 17.152 \\
1.75 & 173 & 1933 & 63.5 & 9.243 & 16.500 \\
2.00 & 166 & 1848 & 69.5 & 8.656 & 15.835 \\
\hline \hline
\end{tabular}


TABLE II. Density dependence of the energies. All energies are given in MeV.

\begin{tabular}{lccccccrr}
\hline \hline$\rho_{B} / \rho_{0}$ & $E_{\sigma}$ & $M_{N}$ & $M^{*}$ & $E_{K}$ & $E_{s}$ & $E_{\omega}$ & $E_{\pi}$ & \multicolumn{1}{c}{$\mathrm{BE}$} \\
\hline 0 & 89.90 & 1241 & 1241 & 1241 & 0 & 0 & 0 & 0 \\
0.25 & 89.89 & 1223 & 1203 & 1210 & 10.15 & 26.56 & -7.431 & -1.729 \\
0.50 & 90.36 & 1197 & 1156 & 1168 & 20.48 & 55.47 & -14.91 & -12.536 \\
0.75 & 90.29 & 1176 & 1112 & 1128 & 31.54 & 86.19 & -19.96 & -15.209 \\
1.00 & 90.25 & 1155 & 1067 & 1087 & 43.24 & 119.2 & -24.02 & -15.717 \\
1.25 & 90.17 & 1138 & 1025 & 1049 & 55.38 & 153.4 & -27.52 & -10.726 \\
1.50 & 90.60 & 1117 & 980.0 & 1008 & 66.40 & 191.3 & -30.58 & -5.701 \\
1.75 & 90.76 & 1097 & 935.9 & 968.8 & 77.72 & 231.4 & -33.33 & 3.278 \\
2.00 & 91.53 & 1078 & 894.0 & 931.5 & 88.42 & 273.6 & -35.83 & 16.432 \\
\hline \hline
\end{tabular}

$$
\mathscr{E}_{\mu}=\rho_{B} E_{\mu},
$$

where the $\mathscr{E}$ 's are given in Eq. (19).

To summarize, our main finding has been to observe that all the relevant masses scale with nuclear-matter density, in a self-consistent calculation. In particular, the scaling $m_{\omega}$ is expected from the missing strength of the longitudinal electron response [27]. We were led to fit nuclear matter more to constrain our model of chiral symmetry breaking. We fit $U(\sigma)$, using the ansatz for the $\sigma$ field given by Eq. (17), in the framework of the relativistic Hartree-Fock calculation [7]. We use the Richardson potential. Calculations with other potentials like, for example, the one suggested by Hansson,
Johnson, and Peterson or Ding, Huang, and Chen [28] and more detailed study of the solutions should be pursued and are under way. We find $M_{N}^{*}$ which are very reasonable and our $\sigma$ is like that of a glueball of mass more than $2 \mathrm{GeV}$. This is similar in spirit to Bayer and Weise [29].

We acknowledge discussions with Professor P. Leal Ferreira and Professor J. Soffer. This work was supported by Conselho Nacional de Desenvolvimento Científico e Tecnológico, by Fundação de Amparo à Pesquisa do Estado de São Paulo (FAPESP), J.D., and also by Grant No. SP/S2/K01/87 from the Department of Science and Technology, Government of India, to M.D.
*On leave from Hoogly Mohsin College, Chinsurah 712101, India.

†On leave at School of Physical Sciences, The Flinders University of South Australia, Bedford Park S.A. 5042, Australia.

†On leave from Instituto de Estudos Avançados, Centro Técnico Aeroespacial, 12231 São José dos Campos, São Paulo, Brazil.

[1] B. L. Ioffe, Nucl. Phys. B118, 317 (1981); B191, 59(E) (1981).

[2] L. J. Reinders, H. R. Rubinstein, and S. Yazaki, Phys. Rep. C 127, 1 (1985).

[3] D. Bailin, J. Cleymans, and M. D. Scadron, Phys. Rev. D 31, 164 (1985).

[4] J. Dey, M. Dey, and P. Ghose, Phys. Lett B 165, 181 (1985).

[5] J. Dey and M. Dey, Phys. Lett. B 176, 469 (1986).

[6] F. E. Close, R. G. Roberts, and G. G. Ross, Phys. Lett. B 168, 400 (1986).

[7] J. Dey, M. Dey, and J. Le Tourneux, Phys. Rev. D 34, 2104 (1986).

[8] G. t'Hooft, Nucl. Phys. B72, 461 (1974).

[9] E. Witten, Nucl. Phys. B160, 57 (1979).

[10] J. Dey, M. Dey, P. Ghose, and G. Mukhopadhyay, Phys. Lett. B 209, 330 (1988).

[11] J. L. Richardson, Phys. Lett. B 82, 272 (1979).

[12] See, e.g, B. D. Serot and J. D. Walecka, The Relativistic Nuclear Many Body Problem, in Advances in Nuclear Phys- ics, Vol. 16, edited by E. Vogt and J. Negele (Plenum, New York, 1986), and references therein.

[13] L. S. Celenza, A. Rozenthal, and C. M. Shakin, Phys. Rev. C 31, 232 (1985).

[14] P. A. M. Guichon, Phys. Lett. B 200, 235 (1988).

[15] T. Frederico, B. V. Carlson, R. A. Rego, and M. S. Hussein, J. Phys. G 15, 297 (1989).

[16] I. Zahed, Phys. Rev. D 30, 2221 (1984).

[17] R. Cenni, F. Conte, and G. Dillon, Lett. Nuovo Cimento 43, 39 (1985).

[18] R. V. Reid, Jr., Ann. Phys. (N.Y.) 50, 411 (1968).

[19] H. W Crater and P. Van Alstine, Phys. Rev. Lett. 53, 1527 (1984).

[20] J. Dey, M. Dey, G. Mukhopadhyay, and B. C. Samanta, Report No. IFT/P-30/89, 1989, São Paulo, Brazil (unpublished).

[21] E. V. Shuryak, The QCD Vacuum, Hadrons and the Superdense Matter (World Scientific, Singapore, 1988).

[22] I. Brevik, Phys. Rev. D 33, 290 (1986).

[23] R. L. Jaffe, Phys. Lett. B 229, 275 (1989). In this paper the $\phi(1030)$ is suggested as a possible second pole in the form factor of the nucleon and correspondingly there is a large $g_{N N \phi}$ coupling implied. Such a coupling was suggested long back in Ref. [25] as Jaffe points out. By implication there is large $\overline{s s}$ component in the nucleon or a violation of the OZI rule [S. Okubo, Phys. Lett. 5, 1975 (1963); G. Zweig, CERN Report No. 8419/Th.412, 1964 (unpublished); J. Iizuka, Progr. Theor. Phys. Suppl. 37-38, 21 
(1966)] for baryons or a combination of both effects.

[24] J. Soffer (private communication). The large $G_{N N \eta}$ suggests the same mechanism as in Ref. [23].

[25] M. M. Nagels, T. A. Rijken, and J. J. de Swart, Phys. Rev. D 12, 744 (1975); D 15, 2547 (1977); 20, 1633 (1979).

[26] R. Friedberg and T. D. Lee, Phys. Rev. D 15, 1694 (1977); 16, 1096 (1977); R. Goldflam and L. Wilets, ibid. 25, 1951 (1982). For a review see L. Wilets, The Nontopological Soliton Model (World Scientific, Singapore, 1989).

[27] G. E. Brown and M. Rho, Phys. Lett. B 222, 324 (1989).

[28] One can have extra parameters to simulate the medium range interpolating between the asymptotic freedom region and confinement in the Richardson potential. This was suggested in T. H. Hansson, K. Johnson, and C. Peterson, Phys. Rev. D 26, 2069 (1989), in their Eq. (48) in a paper where a glueball condensate was suggested as a $\phi$ field, the equivalent of our $\sigma$ field. Another possible unified potential for the meson sector was suggested by $\mathrm{Y}$. Ding, T. Huang, and Z. Chen, Phys. Lett. B 196, 191 (1987).

[29] L. Bayer and W. Weise, Regensburg Report No. TPR-8837. 